Conclusions School district policy combined with promotion of a CAP increased the number of adolescent males who sought out SRH information and condoms from the school nurse. With few points of access to the healthcare system available to males, school nurses may be especially important in connecting males to the healthcare system for services such as STD screening and contraception. As budget shortfalls affect all areas of school administration, school nurses should be recognised and retained as key players in promoting access to SRH services.

\section{5-S1.02 ACCEPTABILITY OF IN SPOT AND PATIENT-DELIVERED PARTNER THERAPY AMONG MEN WHO HAVE SEX WITH MEN SEEKING MEDICAL CARE}

doi:10.1136/sextrans-2011-050109.152

${ }^{1} \mathrm{R}$ Kerani, ${ }^{2} \mathrm{~F}$ Mark, ${ }^{3} \mathrm{M}$ Golden. ${ }^{1}$ Public Health, Seattle \& King County, Seattle, USA; ${ }^{2}$ Public Health, Seattle and King County, USA; ${ }^{3}$ University of Washington, USA

Background Patient-delivered partner therapy (PDPT) and internet partner notification (PN) sites (eg, inSPOT) are being adopted in some areas to improve PN outcomes. Neither intervention has been well-studied in men who have sex with men (MSM). We evaluated the acceptability and potential efficacy of these PN strategies among MSM.

Methods We anonymously surveyed MSM seen in an STD clinic and a private medical practice in Seattle, WA, USA. The survey presented respondents with three scenarios in which they had oral or anal sex with a new partner and were then notified and offered PDPT. A fourth scenario described PN via an inSPOT ecard.

Results MSM completed 198 surveys. A total of 115 (58\%) men reported ever being diagnosed with a bacterial STD, 100 (50\%) had previously been notified by a partner of an STD exposure, and 27 (14\%) reported being HIV positive. The percentage of men who indicated that they would seek medical care was higher when scenarios indicated that the respondent had symptoms of proctitis $(97 \%-98 \%)$ than when they were asymptomatic $(83 \%-89 \%)$ or had symptoms of pharyngitis (84\%). Only 123 (62\%) men said they would seek medical care if notified via an anonymous inSPOT ecard and were asymptomatic, though this rose to $98 \%$ when the question indicated that the respondent had rectal symptoms. Men were somewhat more likely to report that they would take medication given to them as PDPT if they had symptoms than if they were asymptomatic $(52 \%-57 \%$ vs $48 \%-49 \%)$. The proportion of MSM who would seek medical care if asymptomatic was lower among men who said they would take PDPT (74\%-84\%) than among men who indicated that they would not $(92 \%-94 \%, \mathrm{p}<0.06)$; this pattern was not observed when questions described rectal symptoms. When asked directly if they would use inSPOT to notify partners if they had an STD, 56\% said they would. However, when given multiple options and asked how they would prefer to notify partners, only $38 \%$ chose an ecard. MSM were less likely to report that they would seek a medical evaluation if notified via an anonymous ecard than via email or a signed ecard (75\% vs 94\%-95\%).

Conclusions These results suggest that substantial numbers of MSM are interested in using PDPT and internet partner notification sites. However, they also support concerns that PDPT may decrease recipient's likelihood of testing for HIV and syphilis, and that anonymous ecards may be less effective in prompting partners to seek medical care than other forms of PN.

\section{5-S1.03 YOUNG ADULTS' VIEWS ON TELEMEDICINE CONSULTATIONS FOR SEXUAL HEALTH IN AUSTRALIA}

doi:10.1136/sextrans-2011-050109.153

C C Garrett, M Kirkman, J Hocking, M Chen, C K Fairley. University of Melbourne, Australia

Background Young adults in Australia face barriers to accessing sexual health services including concerns over confidentiality and privacy, cost, lack of transportation and limited options around medical providers. A possible solution to decreasing these barriers is the use of telemedicine.

Method An online questionnaire examined young adults' (aged 16-24) views on using webcam and telephone consultations for sexual health in Australia. Descriptive statistics were used to describe the study sample and $\chi^{2}$ was used to assess associations. Free text responses were analysed thematically.

Results 662 people completed the questionnaire. Overall, 23\% $(n=150)$ of participants were willing to have a sexual health consultation with a doctor using a webcam if no genital examination was necessary; this number decreased to $16 \%(n=105)$ if a genital examination over webcam was needed. Men were more willing than women to have a webcam consultation ( $28 \%$ vs $21 \%$, $\mathrm{p}=<0.01$ ). In addition, men with same sex partners were more willing to have webcam consultation, with an unknown doctor, than men without any same sex partners (48\% vs $26 \%, \mathrm{p}=0.04)$. Participants' top preference for consulting a doctor if asymptomatic and living $2 \mathrm{~h}$ from a doctor was telephone $(51 \%, \mathrm{n}=340$, compared with $10 \%$ for webcam); if symptomatic, participants' top preference was in person (62\%, $\mathrm{n}=412$, compared with $16 \%$ for webcam) instead of having the consultation over a webcam. While it was hypothesised that webcam consultations would decrease privacy and confidentiality concerns by preventing people from having to present at a sexual health clinic, preliminary results suggest that webcam consultations may instead augment such concerns. Free text responses suggest that this may be due to the fact that online consultations can be recorded, stored and potentially, if security measures are breached, be retrievable and searchable online.

Conclusion To our knowledge, no study has examined the use of webcam consultations between healthcare providers and clients for sexually transmitted disease care. Results suggest that webcam consultations are not yet an acceptable medium for sexual health consultations for youth in Australia. Concerns about trust, privacy and security around online medical consultations are likely to influence whether such technology is eventually adopted into routine medical care.

\section{5-S1.04 SOCIAL MEDIA AND CHLAMYDIA TESTING BY UNIVERSITY STUDENTS: A PILOT STUDY}

doi:10.1136/sextrans-2011-050109.154

A Norris Turner, T Comston, J Davis, Z Nasrin, J Vaughn. Ohio State University, Columbus, USA

Background Facebook is the world's largest social media site, and university students comprise one of the largest user groups. Sexually transmitted infections are also highly prevalent among university students in the USA. We evaluated a targeted Facebook advertisement for easy, inexpensive chlamydia testing at The Ohio State University (OSU), a public university with 55000 students.

Methods Over 2 weeks in May 2010, our advertisement for \$25 chlamydia testing was displayed to Facebook users who had selfidentified as OSU students between 19 and 28 years of age. Students provided a urine sample directly to the laboratory; a clinician visit was not required. To a separate convenience sample we administered 
a short survey capturing demographics, social media use and interest in future testing.

Results The advertisement received 401732 impressions" (displays of the advertisement to users). Nearly all $98 \%$ ) impressions were to 19-24-year-olds. The daily impressions varied enormously, from 0 to more than 100000 . In the first week, although the advertisement received up to 2,900 daily impressions, no one clicked it. We gradually increased our "bid per click" - the amount we agreed to pay Facebook for each click一from $\$ 0.62$ to $\$ 0.90$. We also modified the advertisement format to depict a college student and to clearly specify the $\$ 25$ cost of the test. Following these changes, both the number of impressions and "clicks" rose dramatically. Seventy-five individuals clicked the advertisement ( 25 women and 50 men), with an average cost per click of $\$ 0.84$. Three students, all female, came for chlamydia testing; one tested positive. Among surveyed individuals $(n=60), 75 \%$ reported using Facebook daily or more often. Few $(10 \%)$ reported ever clicking on Facebook advertisements. Only one surveyed student noticed our Facebook advertisement. The direct-to-laboratory testing concept was popular, with $75 \%$ reporting willingness to use this service in the future.

Conclusions In this short pilot study, 75 individuals clicked on a Facebook advertisement about chlamydia testing, three came for testing and one tested positive. Facebook advertisements are inexpensive, with high exposure to the target population. A small increase in bid per click can make a very large difference in total impressions. The format of the advertisement is critical for catching the attention of the target audience, because many students report never noticing or clicking Facebook advertisements.

\section{5-S1.05 COMPUTER ASSISTED SELF INTERVIEWING IN A SEXUAL HEALTH CLINIC AS PART OF ROUTINE CLINICAL CARE: IMPACT ON SERVICE AND PATIENT AND CLINICIAN VIEWS}

doi:10.1136/sextrans-2011-050109.155

\author{
${ }^{1} \mathrm{~L}$ Vodstrcil, ${ }^{1} \mathrm{~J}$ Hocking, ${ }^{2} \mathrm{R}$ Cummings, ${ }^{1} \mathrm{M}$ Chen, ${ }^{1} \mathrm{C}$ Bradshaw, ${ }^{1} \mathrm{~T}$ Read, ${ }^{2} \mathrm{~J}$ Sze, \\ ${ }^{1} \mathrm{C}$ Fairley. ${ }^{1}$ The University of Melbourne, Parkville, Australia; ${ }^{2}$ Melbourne Sexual Health \\ Centre, Carlton, Australia
}

Background Computer assisted self interviewing (CASI) has been used at the Melbourne Sexual Health Centre (MSHC) in Victoria, Australia since June 2008, to obtain a pre-consultation sexual risk history. We aimed to evaluate the impact of CASI on consultation times, STI testing rates, patient response rates to CASI questions, and obtain patient and clinician views on CASI.

Methods The proportion of patients who declined to answer questions using CASI since 2008 was calculated. We then used the same 12 week period (Feb to May) over three years (2008 pre-CASI), (2009 CASI period), and (2010 non-CASI period-due to a computer theft) to assess consultation times and STI-testing rates. We carried out surveys of clinicians and patients to determine their experience and the acceptability of CASI as part of routine clinical practice.

Results 14190 patients completed CASI during the audit period. Men were more likely than women to decline questions about the number of partners they had of the opposite sex $(4.4 \% \mathrm{v} 3.6 \%$, $p=0.05)$ and same sex $(8.9 \% \vee 0 \%, p<0.01)$. One third $(34 \%)$ of HIVpositive men did not answer questions on number of partners and $18 \%$ declined questions about condom use with insertive anal sex. There was no difference in the mean consultation times during CASI and non-CASI operating periods ( $p$ ?0.17). Only the proportion of women tested for chlamydia differed between CASI and nonCASI periods $(p<0.01,84 \% \vee 88 \%$ respectively). 267 patients completed the survey about CASI. Most $(72 \%$ men and $69 \%$ women) were comfortable using the computer and reported that all their answers were accurate $(76 \%$ men and $71 \%$ women). Half preferred CASI but $18 \%$ would have preferred a clinician to have asked the questions. 39 clinicians completed the staff survey, but a varying proportion $(11 \%-44 \%)$ felt that face-to-face interviewing was more accurate, depending on the risk factor. Only 5\% were unsatisfied with CASI.

Conclusions This is the first evaluation of CASI operating routinely in a sexual health clinic. We have demonstrated that CASI is acceptable to most patients and clinicians in a sexual health setting and does not adversely affect various measures of clinical output. The true value of CASI is most likely to be realised when it is integrated with further innovations in clinical care such as the development of express clinical services, decision support software and detailed behavioural surveillance system.

\section{5-S1.06 STD CLINIC TRIAGE BASED ON COMPUTER-ASSISTED SELF INTERVIEW: THE KING COUNTY EXPERIENCE}

doi:10.1136/sextrans-2011-050109.156

J Dombrowski, M Golden. University of Washington \& Public Health, Seattle \& King County HIVISTD Program, Seattle, USA

Background Triaging patients to different service levels based on responses to computer-assisted self interview (CASI) could increase the cost-efficiency of providing care in STD clinics.

Methods In October 2010 we began having all patients at the King County STD Clinic provide their medical and sexual histories using CASI. Patients are triaged to express or routine care with a computer algorithm based on CASI data. The express care protocol is to collect specimens for HIV/STD testing without physical examination or counselling. Prior to implementation, we conducted 2 months of parallel data collection in which patients who completed CASI were interviewed by clinicians blinded to CASI results. We used $\kappa$ statistics to compare CASI and clinician-obtained data. We tracked visit times for walk-in visits in two 10-day periods pre- and postimplementation, and attempted to survey all patients who used CASI on 10 days about interview and visit type preferences.

Results 875 patients completed both CASI and clinician interviews on 33 days (see Abstract O5-S1.06 table 1). Four months postimplementation, 2731 patients had completed CASI, 420 (15\%) of whom were triaged to express care. Common reasons for triage to routine care were (mutually exclusive): symptoms ( $72 \%$ of routine care), contact to HIV/STD or a symptomatic partner (12\%), and needing a vaccine $(12 \%)$. Patients triaged to express care had lower rates of gonorrhoea $(0.7 \%$ vs $5.7 \%, p<0.001)$, chlamydial infection (3.0\% vs $7.5 \%, p<0.001)$ and syphilis $(0 \%$ vs $1.3 \%, p=0.02)$ than those triaged to routine care. Chart review and informal qualitative data indicated that clinician adherence to the express care protocol varied. Mean visit times were 32 (SD 3) min pre-implementation and 31 (SD 3) min post-implementation. 133 (39\%) of 337 patients completed surveys. $106(80 \%)$ either preferred CASI to clinician interview or had no preference; 27 (59\%) of 46 asymptomatic patients preferred express care.

Conclusions Our CASI system collected accurate data on key aspects of the history, effectively triaged patients at lower risk for bacterial STD diagnosis and was acceptable to most patients surveyed. Lack of acceptability among some clinicians and poor patient recall of vaccine history were barriers to full implementation of the triage system. To date, CASI triage has not improved clinic efficiency as measured by visit time. Our experience suggests that nonclinician staff may be required to implement express care in STD Clinics. 